Supplement of Biogeosciences, 15, 767-780, 2018

https://doi.org/10.5194/bg-15-767-2018-supplement

(C) Author(s) 2018. This work is distributed under

the Creative Commons Attribution 3.0 License.

(c) (1)

Supplement of

\title{
Continuous measurements of nitrous oxide isotopomers during incubation experiments
}

Malte Winther et al.

Correspondence to: Malte Winther (malte.winther@nbi.ku.dk)

The copyright of individual parts of the supplement might differ from the CC BY 3.0 License. 


\section{Supporting material}

\subsection{Oxygen dependence}

The gas matrix of the sample gas is of great importance when using spectroscopy based measuring techniques. When the mixing ratio of the gas matrix is changed, the line shape is also altered, leading to a change in the isotopic signature of the measured sample gas. For the CRDS analyzers, the isotopic signature of the $\mathrm{N}_{2} \mathrm{O}$ isotopologues has a linear response to the oxygen availability in the gas matrix (Erler et al., 2015).

The presented bacterial experiments are performed under anaerobic conditions, where oxygen $\left(\mathrm{O}_{2}\right)$ is not present. The effect of this lack of $\mathrm{O}_{2}$ on the isotopic signal was quantified by performing dilution experiments on the two CIC-MPI standard gasses. Two stepwise dilution measurement with either pure synthetic air or $\mathrm{N}_{2}$ was conducted for each of the standard gases.

10 The $\mathrm{N}_{2}$ was of purity $99.9999 \%$ and the synthetic air was a $\mathrm{N}_{2} / \mathrm{O}_{2}$ mixture $\left(20.1 \% \mathrm{O}_{2}\right.$ and $79.9 \% \mathrm{~N}_{2}$, purity $99.999 \%$ ). Figure $\mathrm{S} 1$ show the average measured $\delta^{15} N^{\alpha}$ values for each step during each of the four four dilution experiments. Similar measurements were performed on $\delta^{15} N^{\beta}$ and $\delta^{15} N^{b u l k}$. The two dilution experiments with synthetic air (Fig. $\mathrm{S} 1 \mathrm{~A}$ and $\mathrm{S} 1 \mathrm{~B}$ ) results in a dependence only on the $\mathrm{N}_{2} \mathrm{O}$ concentration. The two dilution experiments with $\mathrm{N}_{2}$ (Fig. S1C and S1D) results in a dependence on both the $\mathrm{N}_{2} \mathrm{O}$ concentration and the $\mathrm{O}_{2}$ concentration.
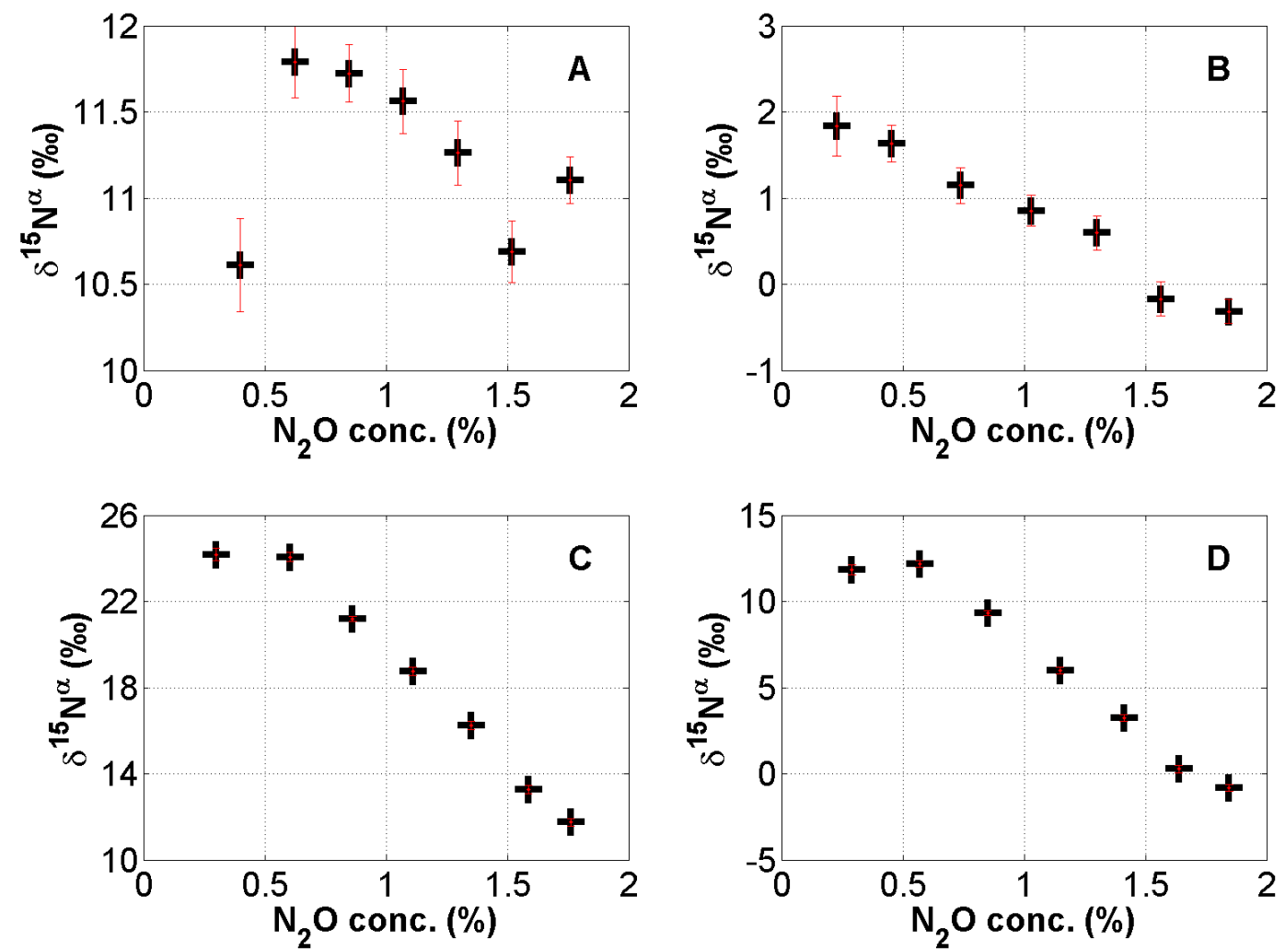

Figure S1. The results of the four dilution experiments for $\delta^{15} N^{\alpha}$. (A) CIC-MPI-II diluted with synthetic air (B) CIC-MPI-I diluted with synthetic air, (C) CIC-MPI-II diluted with $\mathrm{N}_{2}$, and (D) CIC-MPI-I diluted with $\mathrm{N}_{2}$. Black points represent the mean of 30 minutes continuous measurements. The standard error (error bars) of the measurements are shown in red. 
The effect of a changing $\mathrm{O}_{2}$ concentration on the isotopic composition of $\mathrm{N}_{2} \mathrm{O}$ is assessed from the difference between the two dilution experiments for each standard gas. When calculating the difference between the two dilution experiments we isolate the dependence on the $\mathrm{O}_{2}$ concentration, i.e. the difference in $\delta^{15} N^{\alpha}$ is plotted versus the $\mathrm{O}_{2}$ concentration. Figure $\mathrm{S} 2$ show the difference between dilution experiments performed with synthetic air and $\mathrm{N}_{2}$. I.e. the difference between Fig. S1/A 5 and Fig. S1C for CIC-MPI-II and the difference between Fig. S1B and Fig. S1D for CIC-MPI-I. Similar measurements were performed on $\delta^{15} N^{\beta}$ and $\delta^{15} N^{\text {bulk }}$.
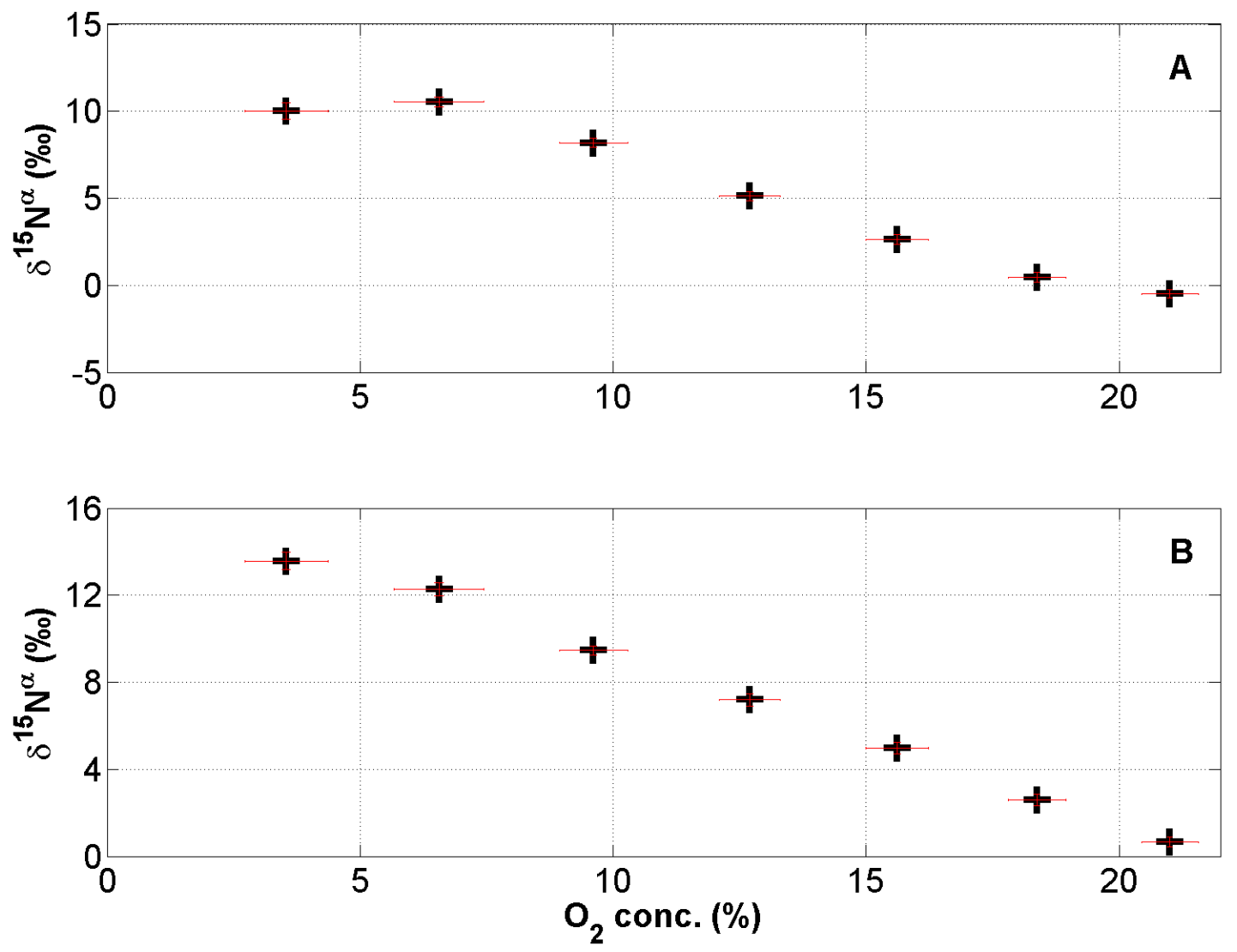

Figure S2. The results of the difference between dilution experiments performed with synthetic air and $\mathrm{N}_{2}$ performed on $\delta^{15} N^{\alpha}$. (A) difference measured on CIC-MPI-I, and (B) difference measured on CIC-MPI-II. Black points represent the mean of 30 minutes continuous measurements. The standard error (error bars) of the measurements are shown in red for both the $\delta$-value and the $\mathrm{O}_{2}$ concentration.

The average response of the isotope composition with respect to the $\mathrm{O}_{2}$ concentration averaged for the two standard gasses are presented in Fig. S3 This relation is calculated using a Monte Carlo algorithm applied to a linear relation model for the $\mathrm{O}_{2}$ concentration and $\delta^{15} N^{\alpha}, \delta^{15} N^{\beta}$, and $\delta^{15} N$ respectively.

10 The linear relation model for the effect of $\mathrm{O}_{2}$ concentration $\left(\left[\mathrm{O}_{2}\right]\right)$ to the values of $\delta^{15} N^{\alpha}, \delta^{15} N^{\beta}$, and $\delta^{15} N$ are shown in equation 1, 2, and 3, respectively.

$\delta^{15} N_{O D C}^{\alpha}=-0.1534 \cdot\left[O_{2}\right]+15.27$ 

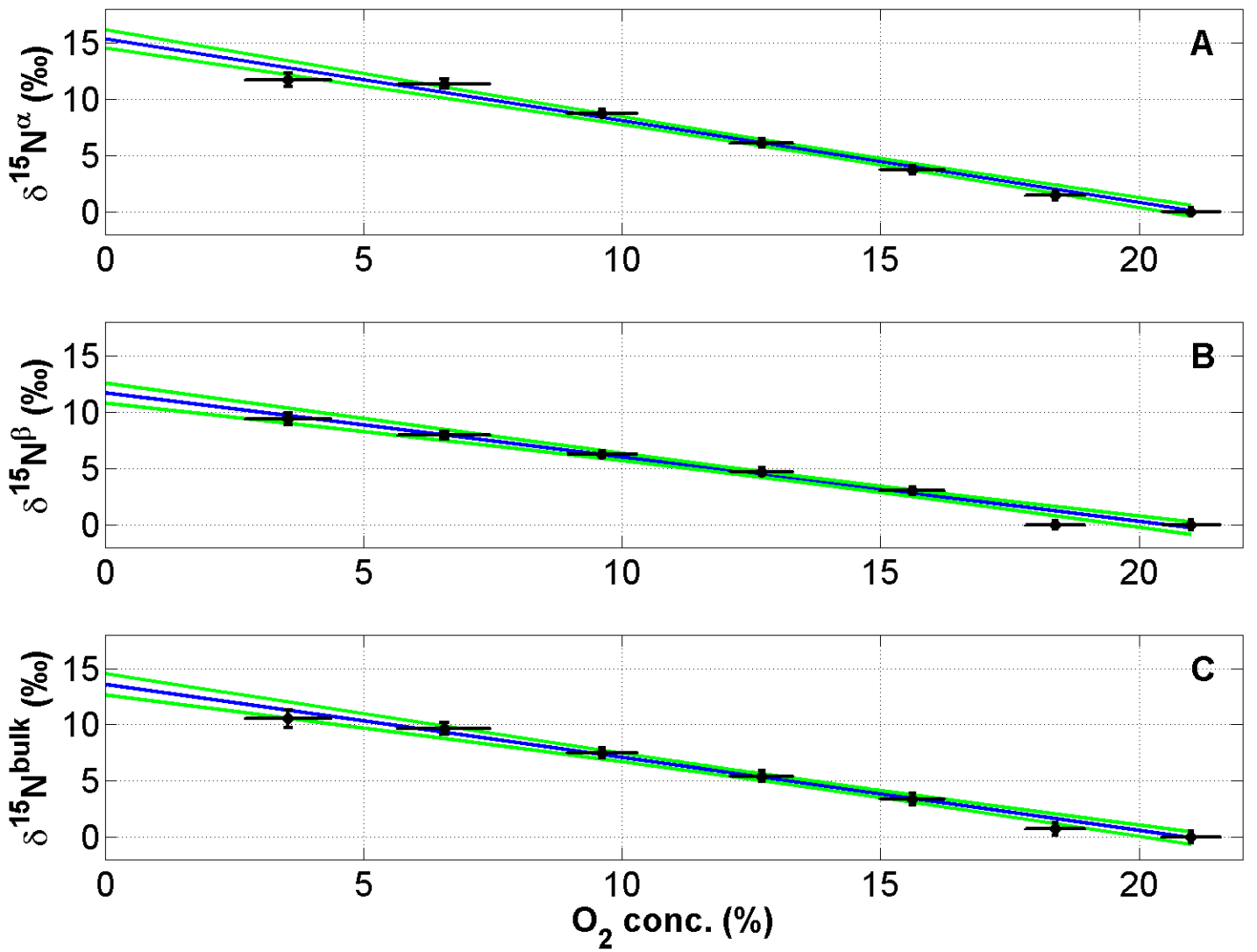

Figure S3. The effect of changing $\mathrm{O}_{2}$ concentration on the $(A) \delta^{15} N^{\alpha}$, (B) $\delta^{15} N^{\beta}$, and $(C) \delta^{15} N$. Black points represent the response of the isotope composition with respect to the $\mathrm{O}_{2}$ concentration averaged for the two standard gasses. The standard error (error bars) of the measurements are shown in black for both the $\delta$-value and the $\mathrm{O}_{2}$ concentration. The blue lines are mean values of the Monte Carlo simulation. The green lines are the 1- $\sigma$ error-bar calculated using Monte Carlo simulation.

$\delta^{15} N_{O D C}^{\beta}=-0.1210 \cdot\left[O_{2}\right]+11.66$

$\delta^{15} N_{O D C}^{\text {bulk }}=-0.1372 \cdot\left[O_{2}\right]+13.47$

5 In the above $\delta^{15} N_{O D C}^{\alpha}, \delta^{15} N_{O D C}^{\beta}$, and $\delta^{15} N_{O D C}^{\text {bulk }}$ represent the offsets which are subtracted from the $\mathrm{N}_{2} \mathrm{O}$ concentration corrected data.

The bacterial evolution experiments were performed under pure $\mathrm{N}_{2}$ conditions. The reported values in the article, are derived by first applying the $\mathrm{N}_{2} \mathrm{O}$ concentration correction and then subsequently applying the correction for the lack of $\mathrm{O}_{2}$. With zero $\mathrm{O}_{2}$ this correction becomes a simple subtraction by the intercept values of the above linear models. 


\subsection{Rayleigh model for isotopomers of $\mathrm{N}_{2} \mathrm{O}$}

Mariotti et al. (1981) derived the equation for the isotope ratio of the substrate as:

$\frac{R_{s}}{R_{s, 0}}=f^{\left(\alpha_{p / s}-1\right)}$

where $R_{s, 0}$ is the initial isotope ratio of the substrate, $R_{s}$ is the isotope ratio of the substrate at time $t, \alpha_{p / s}$ is the fractionation

5 factor of the product versus the substrate, and $f$ is the unreacted fraction of substrate at time $t$. I.e. $f$ is going in steps from 1 to 0 during the reaction. The fractionation factor of the product versus the substrate is the bulk fractionation factor $\alpha_{b u l k}$, and therefore $\alpha_{\text {bulk }}=\left(\alpha_{\alpha}+\alpha_{\beta}\right) / 2$.

The fractionation factor is a constant calculated as $\alpha_{p / s}=R_{p} / R_{s}$. The immediate product for the two isotopomers $\left(R_{i m}^{i}\right)$ therefore calculates as:

$10 R_{i m}^{i}=R_{s} \cdot \alpha_{i}$

$\Uparrow$

$R_{i m}^{i}=\alpha_{i} \cdot R_{s, 0} \cdot f^{\left(\alpha_{b u l k}-1\right)}$

where the isotopomers are distinguished with $i(i=1,2)$, respectively. The accumulated product for each of the isotopomers $\left(R_{p, a c c}^{i}\right)$ can therefore be calculated as the sum of the respective immediate products.

$15 \quad R_{p, a c c}^{i}=\frac{1}{1-f} \int_{f}^{1} R_{i m}^{i} d f^{\prime}$

$\Uparrow$

$R_{p, a c c}^{i}=\frac{1}{1-f} \int_{f}^{1} \alpha_{i} \cdot R_{s, 0} \cdot f^{\prime\left(\alpha_{b u l k}-1\right)} d f^{\prime}$.

where $f$ is the unreacted fraction of the substrate. The equation for the accumulated product for each of the isotopomers derives to:

$R_{p, a c c}^{i}=\alpha_{i} \cdot \frac{R_{s, 0}}{1-f} \int_{f}^{1} f^{\prime\left(\alpha_{b u l k}-1\right)} d f$

$\Downarrow$

$R_{p, a c c}^{i}=\alpha_{i} \cdot \frac{R_{s, 0}}{1-f} \cdot\left[\frac{f^{\prime} \alpha_{b u l k}}{\alpha_{b u l k}}\right]_{f}^{1}$

$\Downarrow$

$R_{p, a c c}^{i}=\frac{\alpha_{i}}{\alpha_{b u l k}} \cdot R_{s, 0} \cdot \frac{1-f^{\alpha_{b u l k}}}{1-f}$

25

$\Downarrow$

$R_{p, a c c}^{i}=\frac{\alpha_{i}}{\alpha_{b u l k}} \cdot R_{p, a c c}^{b u l k}$.

The isotopomer correction factors for the two isotopomers $\left(\varphi_{\alpha}\right.$ and $\left.\varphi_{\beta}\right)$ therefore ends up as presented in the manuscript.

$\varphi_{\alpha}=\frac{\alpha_{\alpha}}{\alpha_{b u l k}}, \varphi_{\beta}=\frac{\alpha_{\beta}}{\alpha_{b u l k}}$ 


\subsection{Iterative determination of unreacted fraction $(f)$}

\subsubsection{Figures of $f_{\text {start }}$ for $\delta^{15} N^{\alpha}$}

Figures of the iterative determination of the starting point of the unreacted fraction $(f)$ of $\delta^{15} N^{\alpha}$ versus the calculated Rsquared value. The black crosses are all possible $f$-values used in calculation of $\mathbf{R}^{2}$ between the Rayleigh fractionation profile

5 and the measured data produced from Pseudomonas fluorescens. The red crosses are the best fit to $\delta^{15} N^{\alpha}$. The green crosses are the average best fit to $\delta^{15} N^{\alpha}$ and $\delta^{15} N^{\beta}$, hence the used values. Figure $\mathrm{A}$ is the first replica and the one presented in the manuscript.
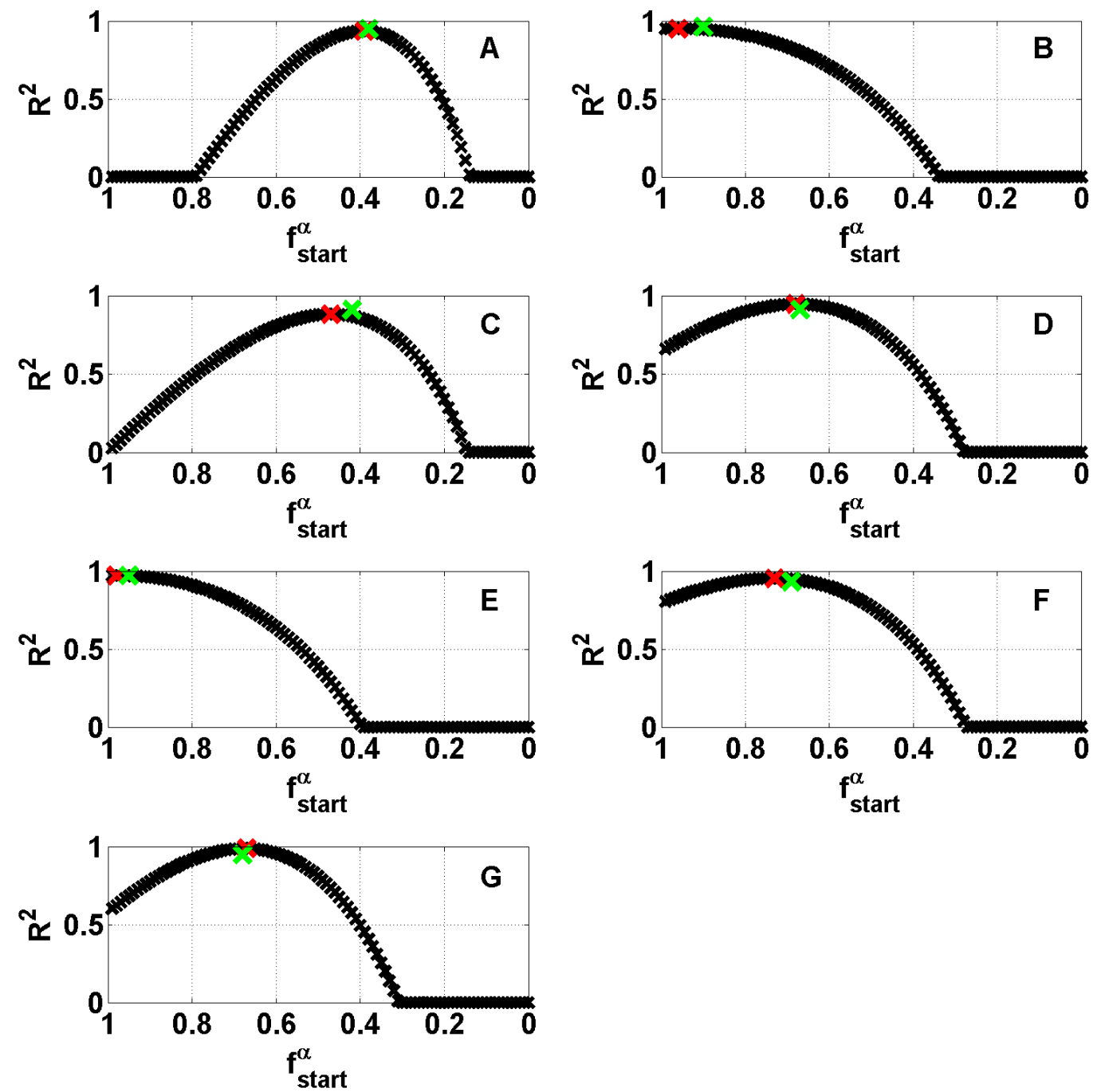


\subsubsection{Figures of $f_{\text {start }}$ for $\delta^{15} N^{\beta}$}

Figures of the iterative determination of the starting point of the unreacted fraction $(f)$ of $\delta^{15} N^{\beta}$ versus the calculated Rsquared value. The black crosses are all possible $f$-values used in calculation of $\mathbf{R}^{2}$ between the Rayleigh fractionation profile and the measured data produced from Pseudomonas fluorescens. The red crosses are the best fit to $\delta^{15} N^{\beta}$. The green crosses

5 are the average best fit to $\delta^{15} N^{\alpha}$ and $\delta^{15} N^{\beta}$, hence the used values. Figure $\mathrm{A}$ is the first replica and the one presented in the manuscript.
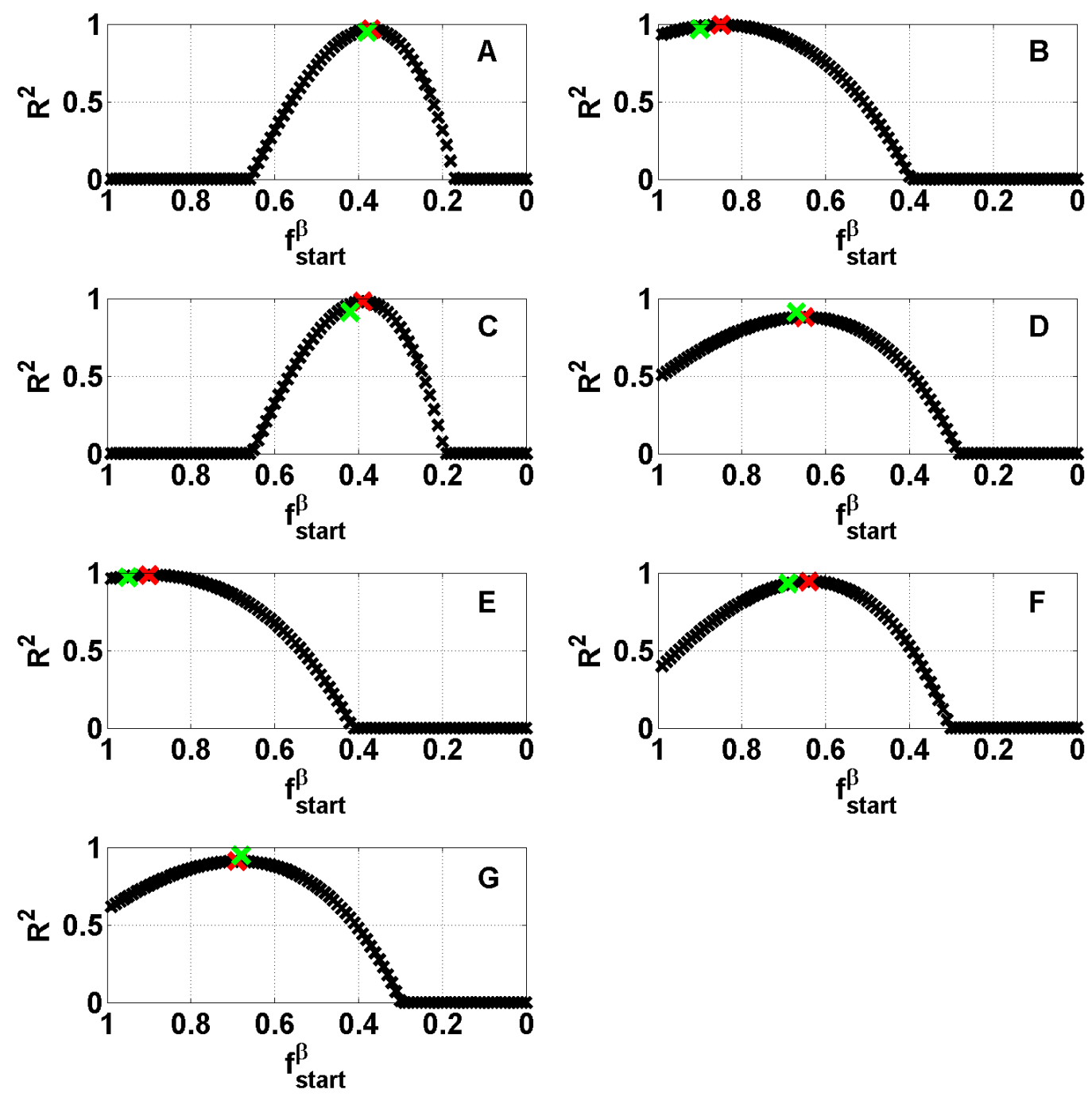


\subsubsection{Figures of $f_{\text {end }}$}

Figures of the iterative determination of the ending point of the unreacted fraction $(f)$ versus the calculated R-squared value. The black crosses are all possible $f$-values used in calculation of $\mathbf{R}^{2}$ between the Rayleigh fractionation profile and the measured data produced from Pseudomonas fluorescens. The green crosses are the best fit to $\delta^{15} N^{\alpha}$ and $\delta^{15} N^{\beta}$, hence the used values. Figure $\mathrm{A}$ is the first replica and the one presented in the manuscript.
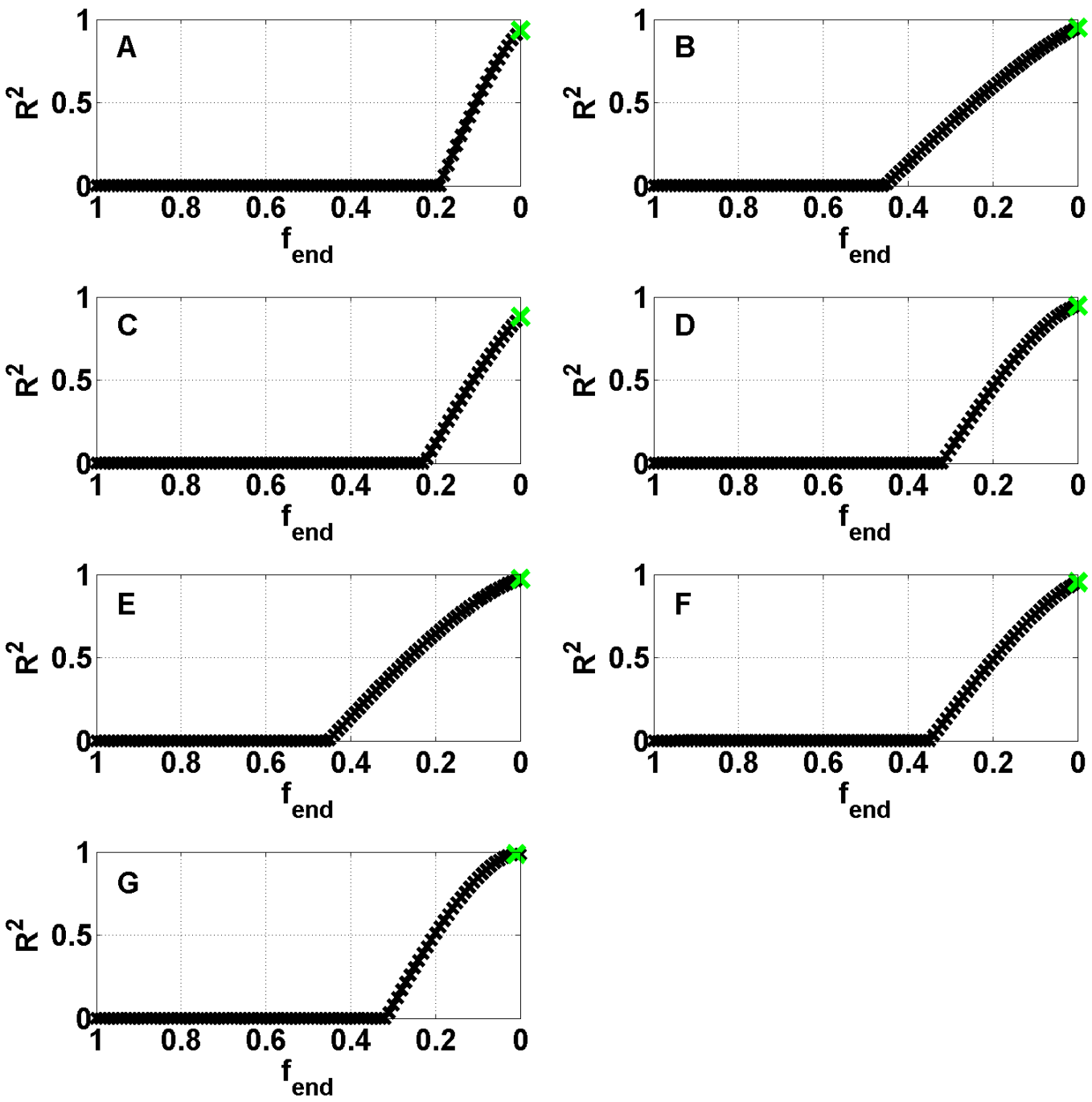


\subsection{Iterative determination of the reduction correction parameter $(\gamma)$}

Figures of the iterative determination of the starting point of the reduction correction parameter $(\gamma)$ versus the calculated Rsquared value. The black crosses are all possible $\gamma$-values used in calculation of $\mathrm{R}^{2}$ between the Rayleigh fractionation profile and the measured data produced from Pseudomonas fluorescens. The green crosses are the best fit to $\delta^{15} N^{\alpha}$ and $\delta^{15} N^{\beta}$, hence the used values. Figure A is the first replica and the one presented in the manuscript.
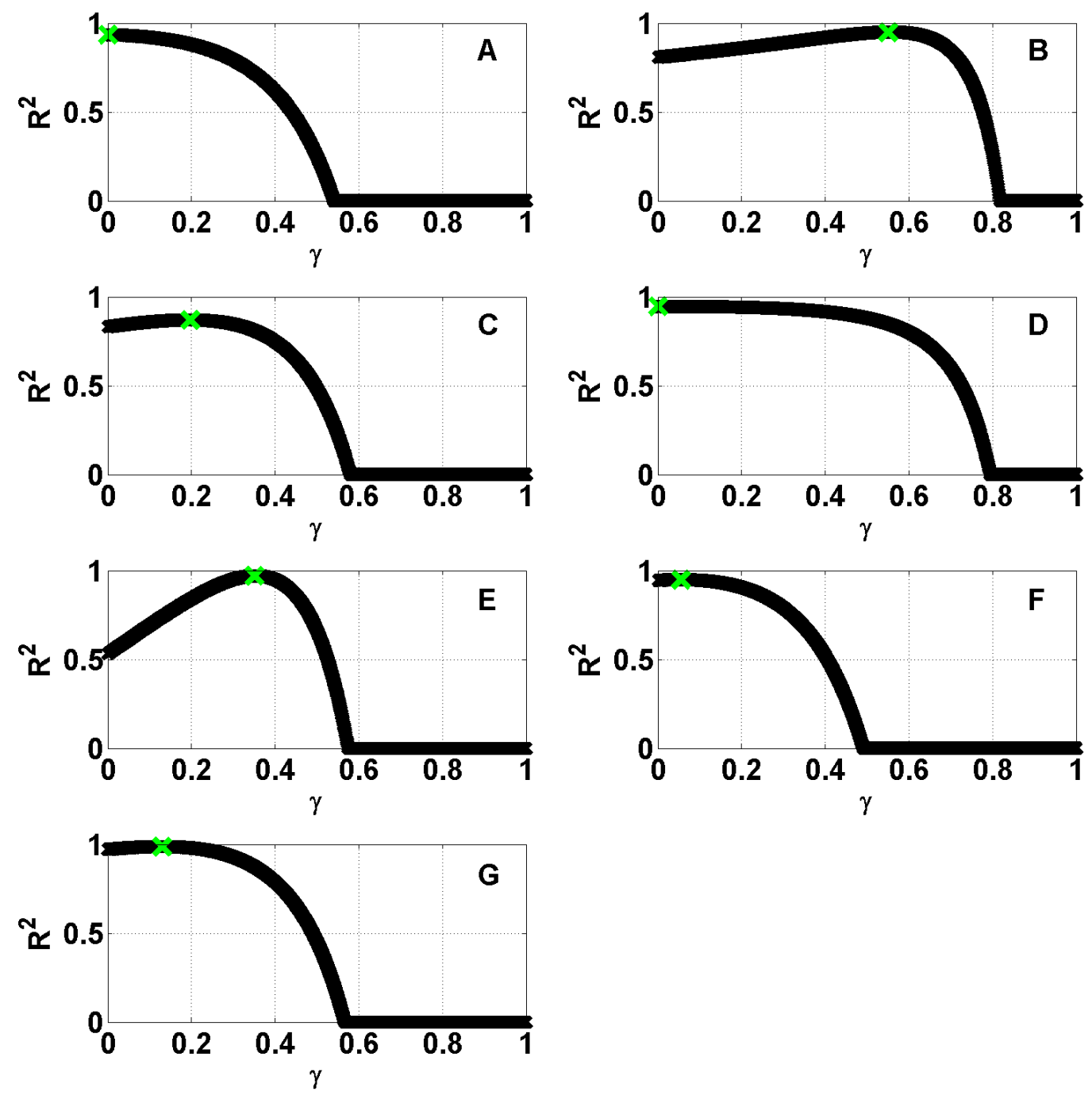


\subsection{Pseudomonas Chlororaphis}

\subsubsection{Figures of $\delta^{15} N^{\alpha}$}

Figures of the continuous measurements of the evolution of $\delta^{15} N^{\alpha}$ versus the concentration of $\mathrm{N}_{2} \mathrm{O}$. The blue profile is the raw production part. The black profile is the five minutes running mean of the raw measurements. The red is the fitted Rayleigh distillation for the production part. Figure $\mathrm{A}$ is the first replica and the one presented in the manuscript.
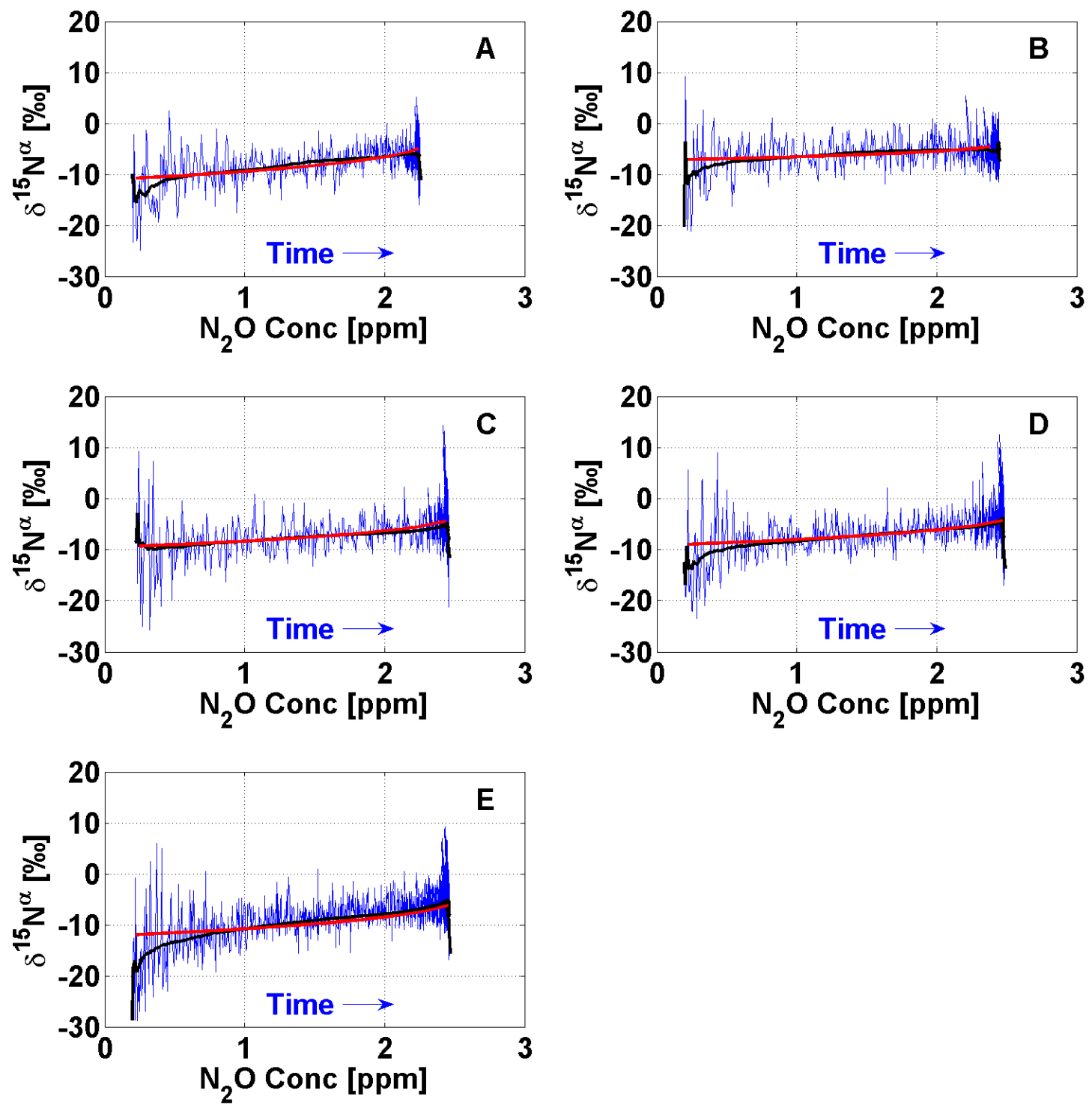


\subsubsection{Figures of $\delta^{15} N^{\beta}$}

Figures of the continuous measurements of the evolution of $\delta^{15} N^{\beta}$ versus the concentration of $\mathrm{N}_{2} \mathrm{O}$. The blue profile is the raw production part. The black profile is the five minutes running mean of the raw measurements. The red is the fitted Rayleigh distillation for the production part. Figure $\mathrm{A}$ is the first replica and the one presented in the manuscript.
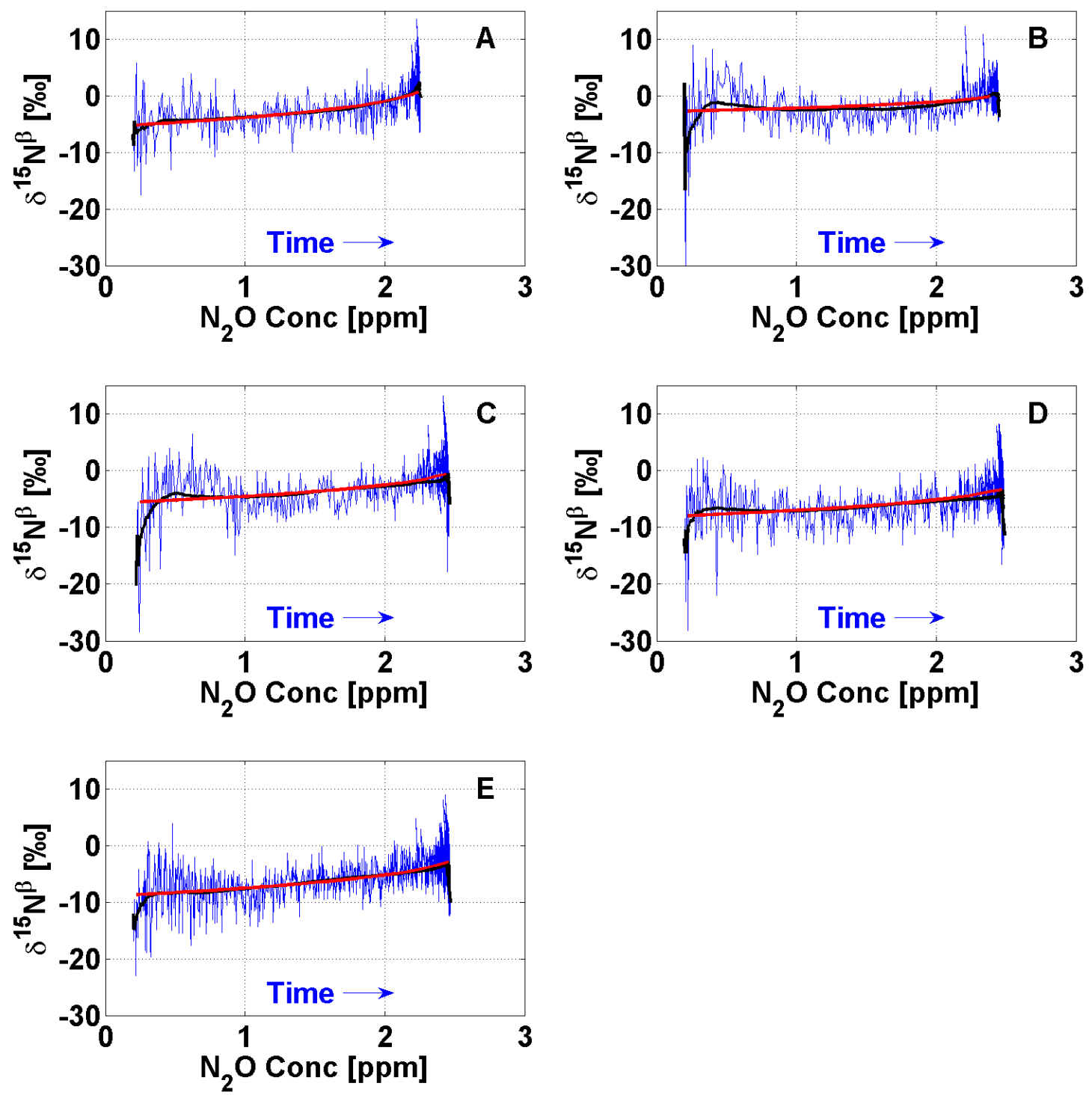


\subsubsection{Figures of $\delta^{15} N^{b u l k}$}

Figures of the continuous measurements of the evolution of $\delta^{15} N^{b u l k}$ versus the concentration of $\mathrm{N}_{2} \mathrm{O}$. The blue profile is the raw production part. The black profile is the five minutes running mean of the raw measurements. The red is the fitted Rayleigh distillation for the production part. Figure $\mathrm{A}$ is the first replica and the one presented in the manuscript.
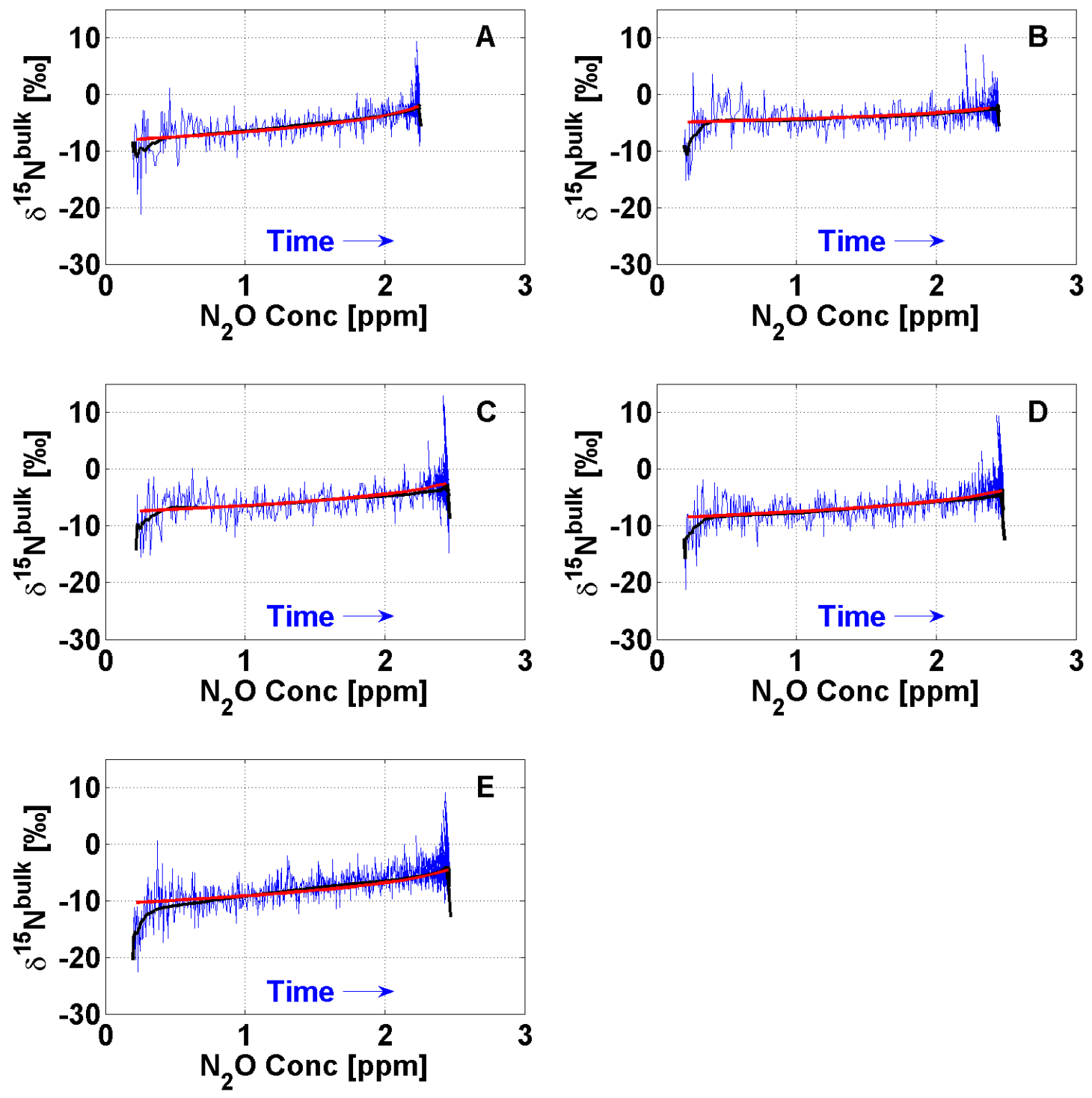


\subsubsection{Figures of SP}

Figures of the continuous measurements of the evolution of SP versus the concentration of $\mathrm{N}_{2} \mathrm{O}$. The blue profile is the raw production part. The black profile is the five minutes running mean of the raw measurements. Figure $\mathrm{A}$ is the first replica and the one presented in the manuscript.
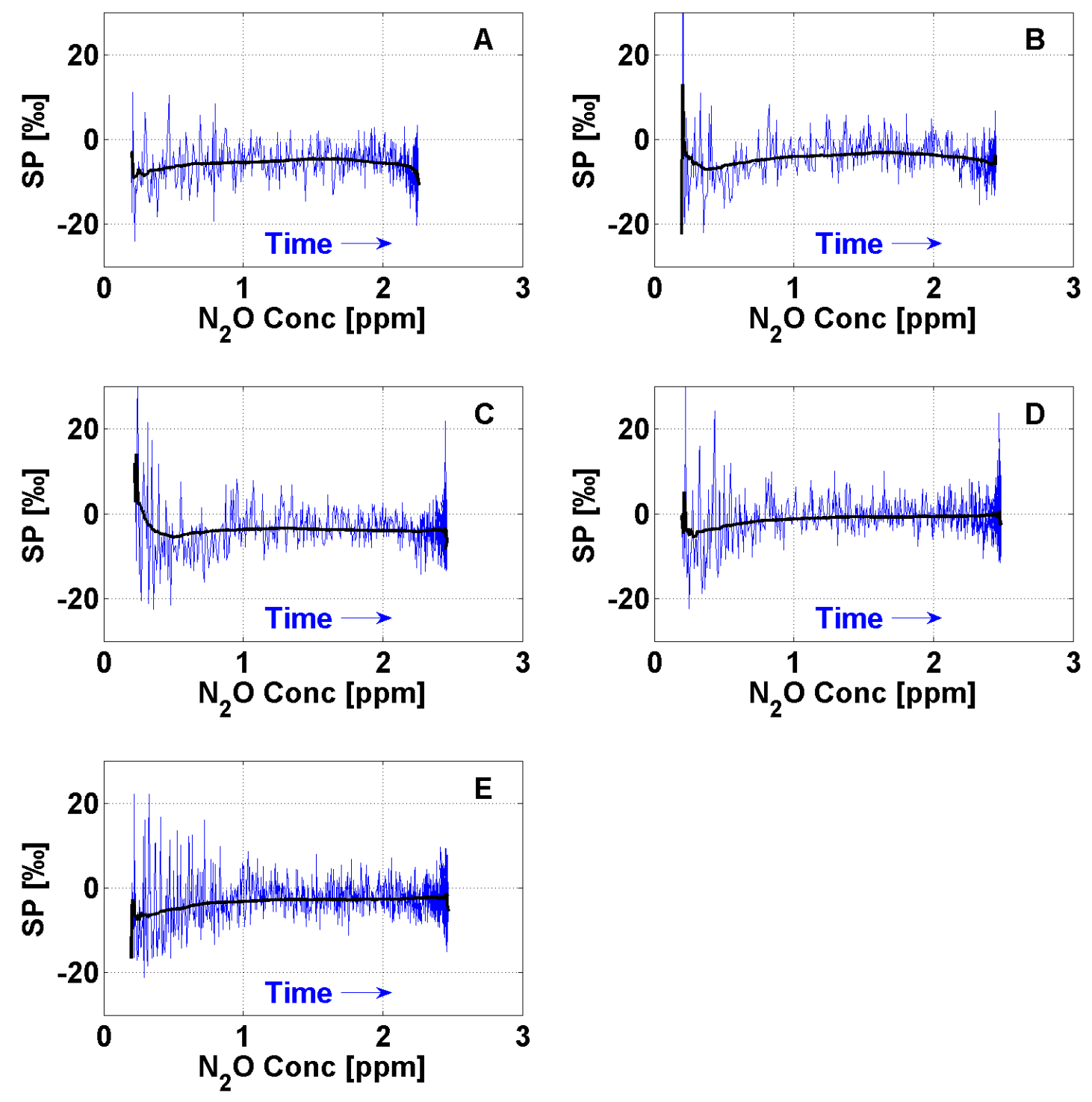


\subsection{Pseudomonas Fluorescens}

\subsubsection{Figures of $\delta^{15} N^{\alpha}$}

Figures of the continuous measurements of the evolution of $\delta^{15} N^{\alpha}$ versus the concentration of $\mathrm{N}_{2} \mathrm{O}$. The blue profile is the raw production part. The green profile is the raw consumption part. The black profile is the 5 minutes running mean of the raw

5 measurements. The red is the fitted Rayleigh distillation for the production part. The magenta is the fitted Rayleigh distillation for the consumption part. Figure $\mathrm{A}$ is the first replica and the one presented in the manuscript.
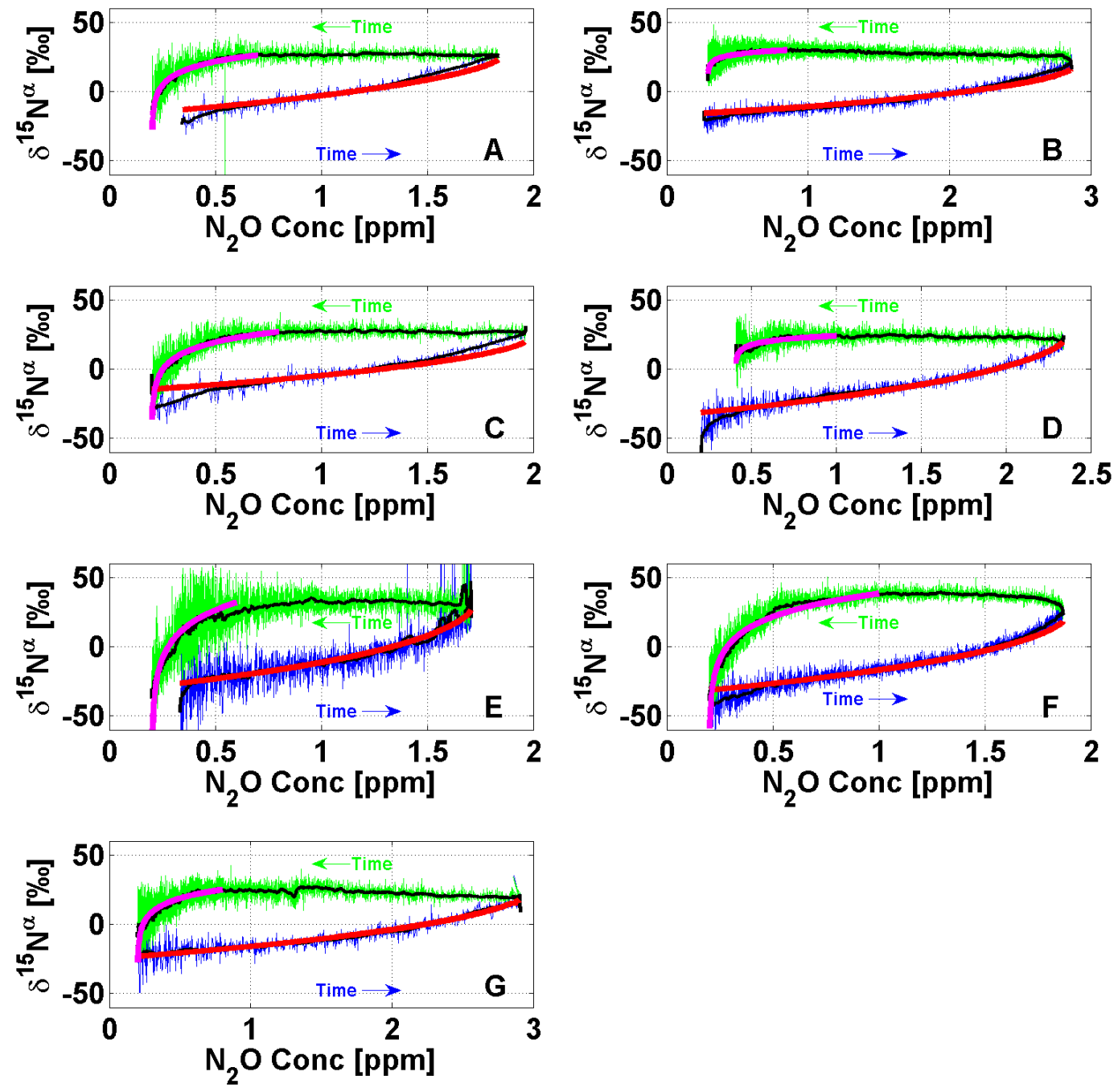


\subsubsection{Figures of $\delta^{15} N^{\beta}$}

Figures of the continuous measurements of the evolution of $\delta^{15} N^{\beta}$ versus the concentration of $\mathrm{N}_{2} \mathrm{O}$. The blue profile is the raw production part. The green profile is the raw consumption part. The black profile is the five minutes running mean of the raw measurements. The red is the fitted Rayleigh distillation for the production part. The magenta is the fitted Rayleigh distillation for the consumption part. Figure A is the first replica and the one presented in the manuscript.
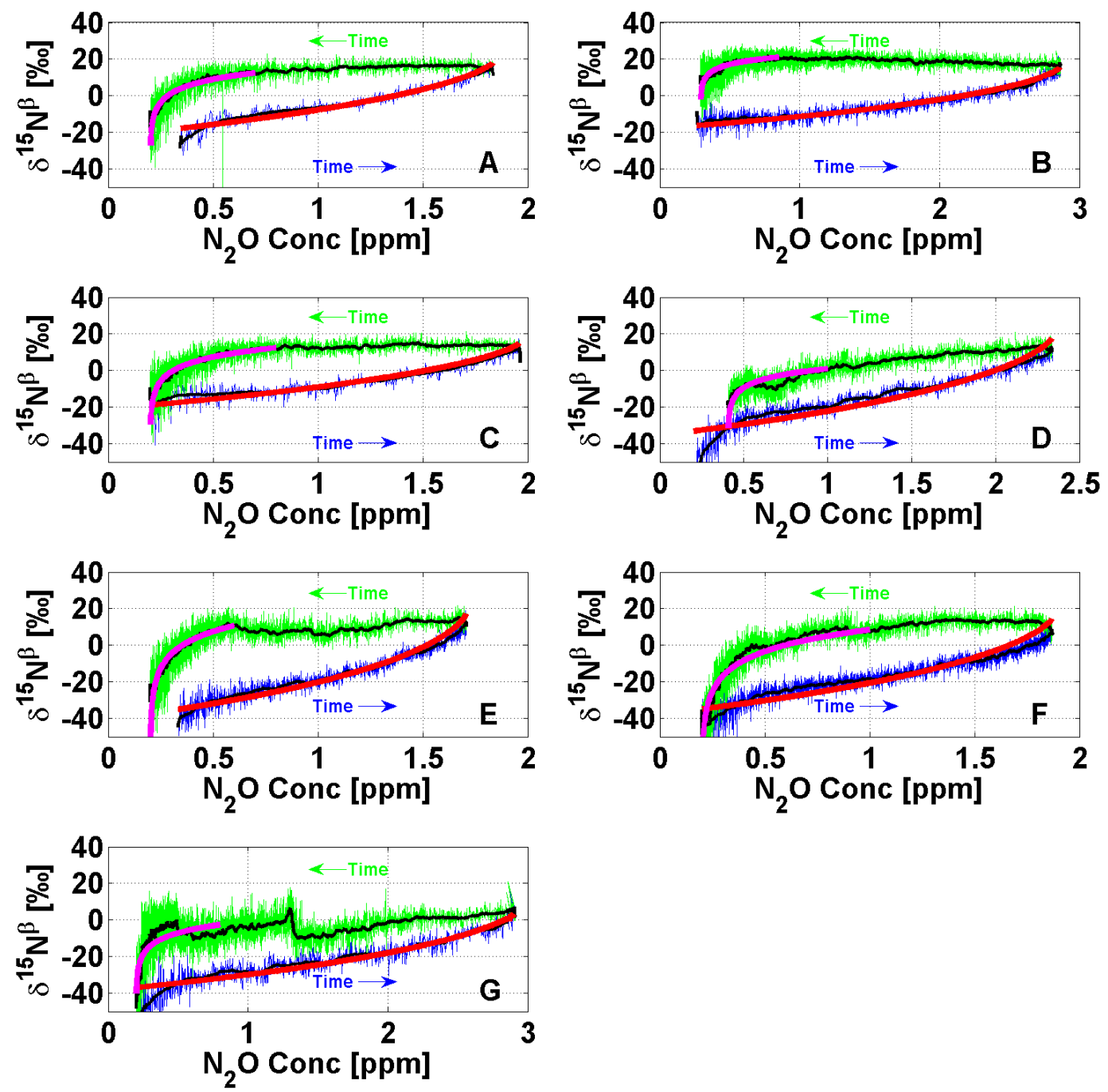


\subsubsection{Figures of $\delta^{15} N^{b u l k}$}

Figures of the continuous measurements of the evolution of $\delta^{15} N^{b u l k}$ versus the concentration of $\mathrm{N}_{2} \mathrm{O}$. The blue profile is the raw production part. The green profile is the raw consumption part. The black profile is the five minutes running mean of the raw measurements. The red is the fitted Rayleigh distillation for the production part. The magenta is the fitted Rayleigh distillation for the consumption part. Figure $\mathrm{A}$ is the first replica and the one presented in the manuscript.
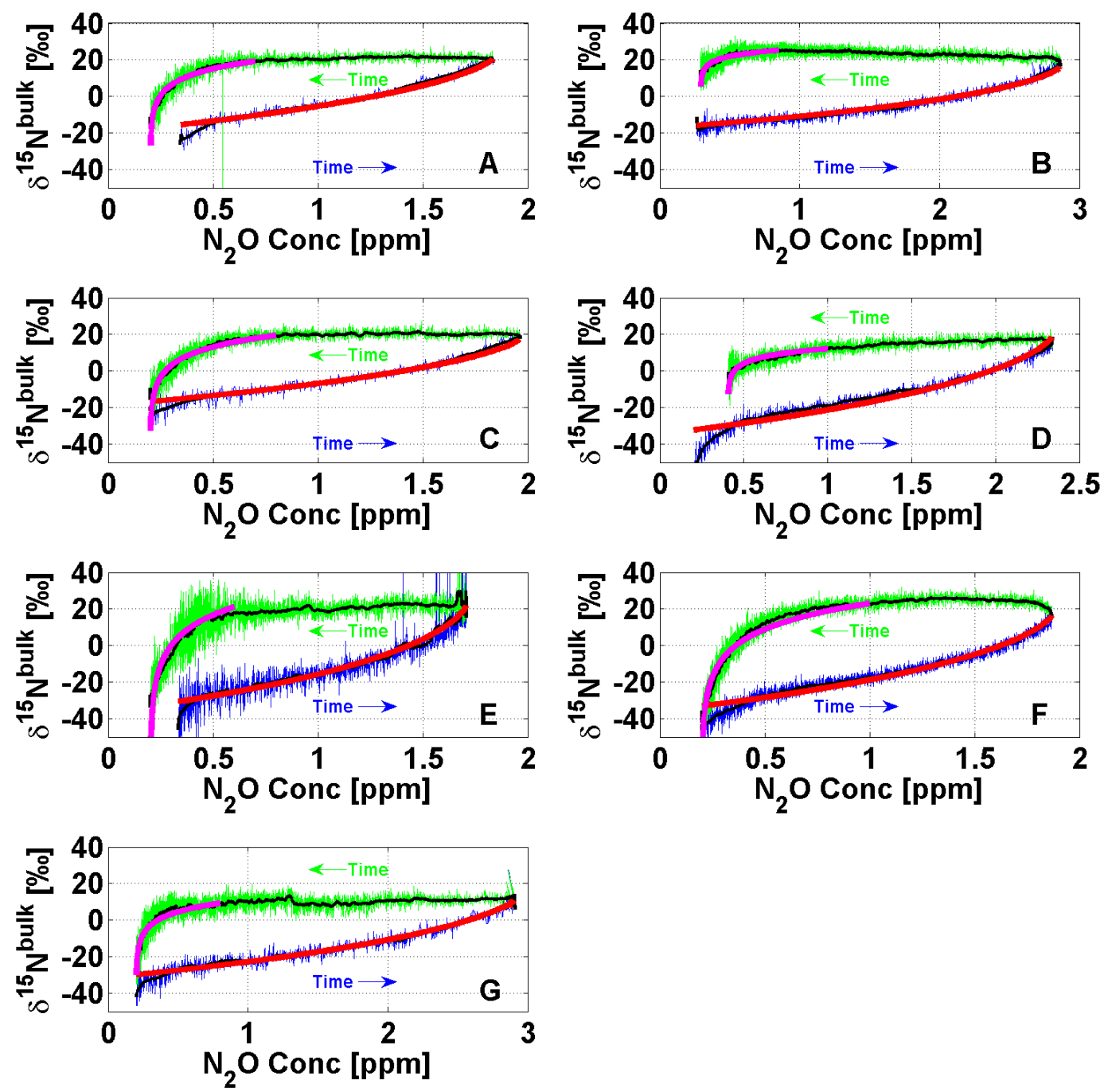


\subsubsection{Figures of SP}

Figures of the continuous measurements of the evolution of SP versus the concentration of $\mathrm{N}_{2} \mathrm{O}$. The blue profile is the raw production part. The green profile is the raw consumption part. The black profile is the five minutes running mean of the raw measurements. Figure A is the first replica and the one presented in the manuscript.
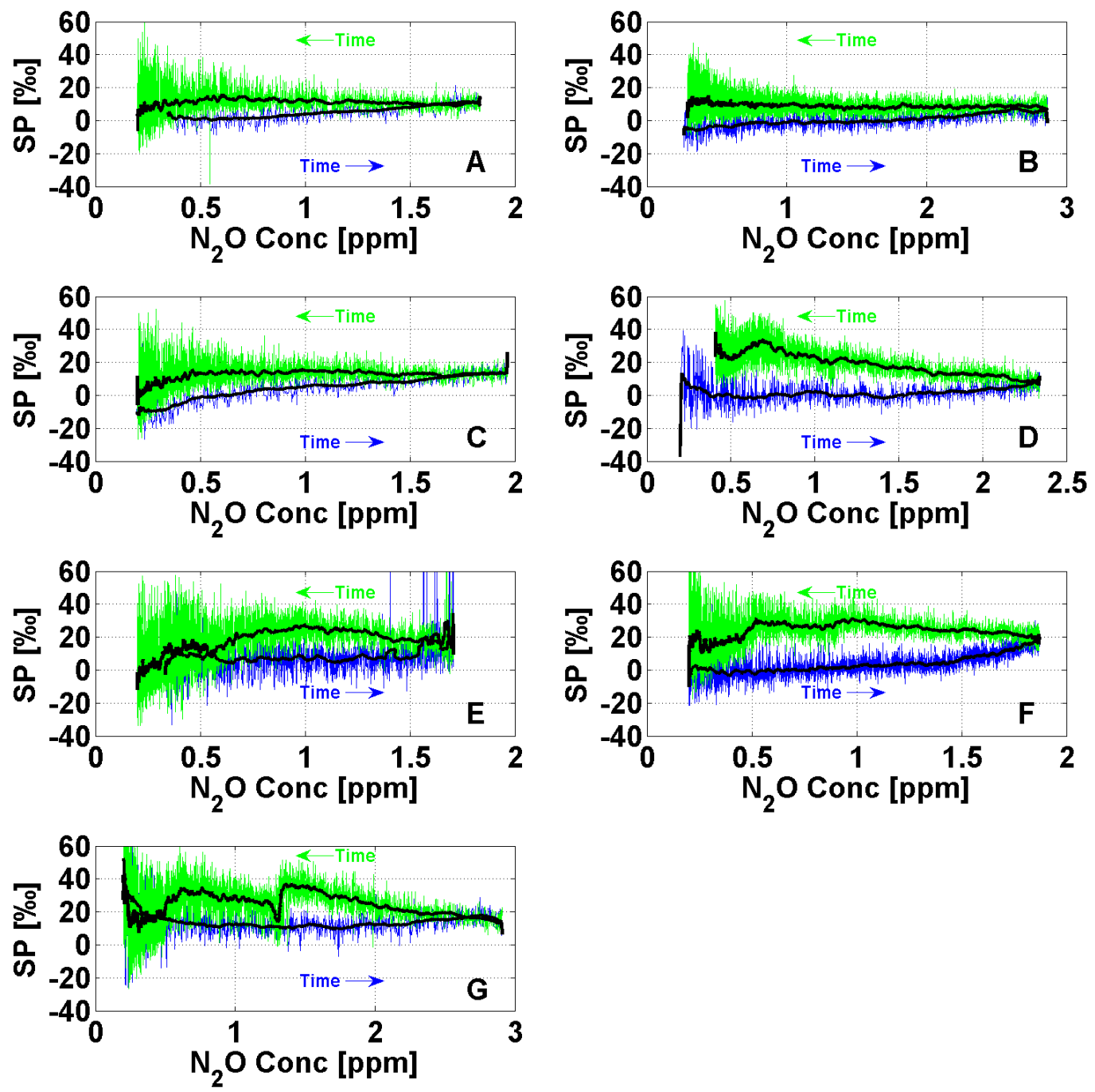